\title{
Unravelling the adaptation responses to osmotic and temperature stress in Chromohalobacter salexigens, a bacterium with broad salinity tolerance
}

\author{
Carmen Vargas, Montserrat Argandoña, Mercedes Reina-Bueno, \\ Javier Rodríguez-Moya, Cristina Fernández-Aunión and Joaquín J Nieto*
}

Address: Department of Microbiology and Parasitology, Faculty of Pharmacy, University of Seville, 41012 Seville, Spain

Email: Carmen Vargas - cvargas@us.es; Montserrat Argandoña - montseab@us.es; Mercedes Reina-Bueno - mrb@us.es; Javier RodríguezMoya - jrmv@us.es; Cristina Fernández-Aunión - c-aunion@us.es; Joaquín J Nieto* - jjnieto@us.es

* Corresponding author

Published: 15 September 2008

Saline Systems 2008, 4:14 doi:10.1186/1746-1448-4-14

This article is available from: http://www.salinesystems.org/content/4/l/14

(C) 2008 Vargas et al; licensee BioMed Central Ltd.

This is an Open Access article distributed under the terms of the Creative Commons Attribution License (http://creativecommons.org/licenses/by/2.0), which permits unrestricted use, distribution, and reproduction in any medium, provided the original work is properly cited.
Received: 2 April 2008

Accepted: I5 September 2008

\begin{abstract}
Chromohalobacter salexigens, a Gammaproteobacterium belonging to the family Halomonadaceae, shows a broad salinity range for growth. Osmoprotection is achieved by the accumulation of compatible solutes either by transport (betaine, choline) or synthesis (mainly ectoine and hydroxyectoine). Ectoines can play additional roles as nutrients and, in the case of hydroxyectoine, in thermotolerance. A supplementary solute, trehalose, not present in cells grown at $37^{\circ} \mathrm{C}$, is accumulated at higher temperatures, suggesting its involvement in the response to heat stress. Trehalose is also accumulated at $37^{\circ} \mathrm{C}$ in ectoine-deficient mutants, indicating that ectoines suppress trehalose synthesis in the wild-type strain. The genes for ectoine (ectABC) and hydroxyectoine (ectD, ectE) production are arranged in three different clusters within the $C$. salexigens chromosome. In order to cope with changing environment, $C$. salexigens regulates its cytoplasmic pool of ectoines by a number of mechanisms that we have started to elucidate. This is a highly complex process because (i) hydroxyectoine can be synthesized by other enzymes different to EctD (ii) ectoines can be catabolized to serve as nutrients, (iii) the involvement of several transcriptional regulators $\left(\sigma^{S}, \sigma^{32}\right.$, Fur, EctR) and hence different signal transduction pathways, and (iv) the existence of post-trancriptional control mechanisms. In this review we summarize our present knowledge on the physiology and genetics of the processes allowing $C$. salexigens to cope with osmotic stress and high temperature, with emphasis on the transcriptional regulation.
\end{abstract}

\section{Background}

The availability of water in their habitat is one of the most important parameters affecting the survival and growth of microorganisms. Thus, they must be able to cope and adapt to the frequent fluctuations in the water content of the external environment to maintain their turgor pressure within limits necessary for growth and multiplication. Osmoadaptation mechanisms are referred as those physiological and genetic manifestations of adaptation to low and high water environments [1]. Prokaryotic osmoadaptation has recently gained considerable importance not only to understand how bacterial cells cope with environmental conditions but also because this knowledge can be applied in agriculture, food and fermentation industries, and biomedicine [2]. 
The intracellular accumulation of large quantities of a particular group of small, organic osmolytes is a common and flexible strategy of osmoadaptation for eukaryotic and prokaryotic microorganisms when they need to cope with hyperosmotic conditions. These compounds, which function as osmoprotectants, are termed compatible solutes since they can be amassed by the cell in very high concentrations providing osmotic balance without disturbing essential cellular functions and the correct folding of proteins [3]. Since the cell may also release these solutes upon a hypoosmotic shock by using specific efflux systems restoring the osmotic balance, this strategy enables organisms to adapt to a wide range of salt concentrations by adjusting the cytoplasmic solute pool to the osmolality of the surrounding environment. For this reason, it is not surprising that this is an evolutionary well-conserved adaptation strategy in a wide diversity of microorganisms [4-6]. Although there is a relatively high diversity of compounds reported as compatible solutes, they are mainly sugars (sucrose and trehalose), polyols (glycerol, glucosylglycerol, mannosylglycerol, and arabitol, among others), amino acids (glutamine and derivatives, proline, alanine), quaternary amines (betaines, choline) and ectoines (ectoine and $\beta$-hydroxyectoine). Sometimes they are accumulated after their transport when present in the medium or, in some cases, an external precursor is taken up and later on it is converted into the specific compatible solute. Other compounds can only be accumulated after their synthesis de novo, by using a specific biosynthetic pathway, i.e., when cells are growing in a minimal medium. In any case, it is generally assumed that uptake of external osmoprotectants in the medium is energetically preferred over synthesis de novo [4,7-9]. Recently there is an increasing body of evidence indicating that compatible solutes are multifunctional molecules with stabilizing properties, hence protecting cell components from several abiotic stresses such as high salinity, freezing, desiccation, high temperature, pressure, or oxygen radicals, functioning as chemical chaperones [10]. This multistress protection is especially remarkable in the case of some of them, such as trehalose and ectoines [11,12], which have current and potential applications in molecular biology, agriculture, biotechnology and biomedicine $[13,14]$. In some circumstances, some of these osmoprotectants can be also used as efficient carbon and nitrogen sources, such as the ectoines or betaines, which are widespread in nature $[9,15]$.

Moderately halophilic bacteria (growing optimally between 0.5 and $2.5 \mathrm{M}$ salt) [16] of the family Halomonadaceae, such as Halomonas elongata and Chromohalobacter salexigens (formerly H. elongata DSM 3043) [17], show a remarkable versatility with respect to their salt tolerance and have been extensively used in recent years to study the bacterial osmoadaptation processes [16]. More than a decade ago, we selected C. salexigens as a model organism for such studies due to the following reasons. First, it has an unusual extremely broad salinity range, one of the widest found in Nature, growing in the presence of $\mathrm{NaCl}$ concentrations ranging from ca. 0.1 to $4 \mathrm{M}$ in complex medium in a temperature range of 15 to $45^{\circ} \mathrm{C}$ [17], and from 0.5 to $3 \mathrm{M}$ in minimal medium M63, with optimal growth at $1.5 \mathrm{M} \mathrm{NaCl}$ and $37^{\circ} \mathrm{C} \mathrm{[18].} \mathrm{It} \mathrm{is} \mathrm{worth} \mathrm{mention-}$ ing that $C$. salexigens is a true halophile, requiring at least $0.5 \mathrm{M} \mathrm{NaCl}$ for any growth at all in minimal medium [18]. Interestingly, $0.5 \mathrm{M} \mathrm{NaCl}$ is the maximal salt concentration that the non halophilic E. coli, traditionally used for osmoregulation studies, can tolerate [1]. It has been recently reported that while $C$. salexigens needs moderate concentrations of $\mathrm{Na}^{+}$and $\mathrm{Cl}^{-}$ions, its growth rate was stimulated by a number of other salts, indicating that it requires a combination of $\mathrm{NaCl}$ and high ionic strength for optimal growth [19]. Secondly, C. salexigens is a metabolically versatile bacterium, growing fast on a wide range of simple carbon compounds as its sole carbon and energy source [17]. Another advantage is that tools that allow the genetic manipulation of $C$. salexigens have been largely developed [20]. Finally, the C. salexigens complete genome sequence has recently been determined by the Joint Genome Institute (an automatically generated annotation is available at http://genome.ornl.gov/microbial/ csal/), enabling an in silico search for systems involved in halophilic behaviour and halotolerance.

Although adaptive changes in its membrane lipid composition contribute to the long-term response of $C$. salexigens to salt stress [21], osmoadaptation is mainly achieved upon accumulation of compatible solutes, after their de novo synthesis or by transport from the surrounding media when available. The main endogenous compatible solutes are ectoines (ectoine and hydroxyectoine) and a mutant unable to synthesize them cannot grow above $0.75 \mathrm{M} \mathrm{NaCl}$ in a minimal medium such as M63, in contrast to the wild type strain, which grows well up to $3 \mathrm{M}$ $\mathrm{NaCl}$ in the same medium [22]. Here we summarize our present knowledge on the response of $C$. salexigens to high salinity and high temperature. Our data show that the synthesis of ectoines by this extremophile is a highly complex process that is both osmo- and thermoregulated, and controlled at both the transcriptional and post-trancriptional level.

\section{Uptake of osmoprotectants from environmental sources}

C. salexigens accumulates externally supplied glycine betaine (named betaine hereafter) either by direct transport or after oxidation of its precursor choline in a twostep enzymatic pathway that has been characterized at the biochemical and molecular level (Fig. 1) [23,24]. C. salexigens genome carries several set of genes that may encode ABC-type betaine uptake systems (see below), but none of 


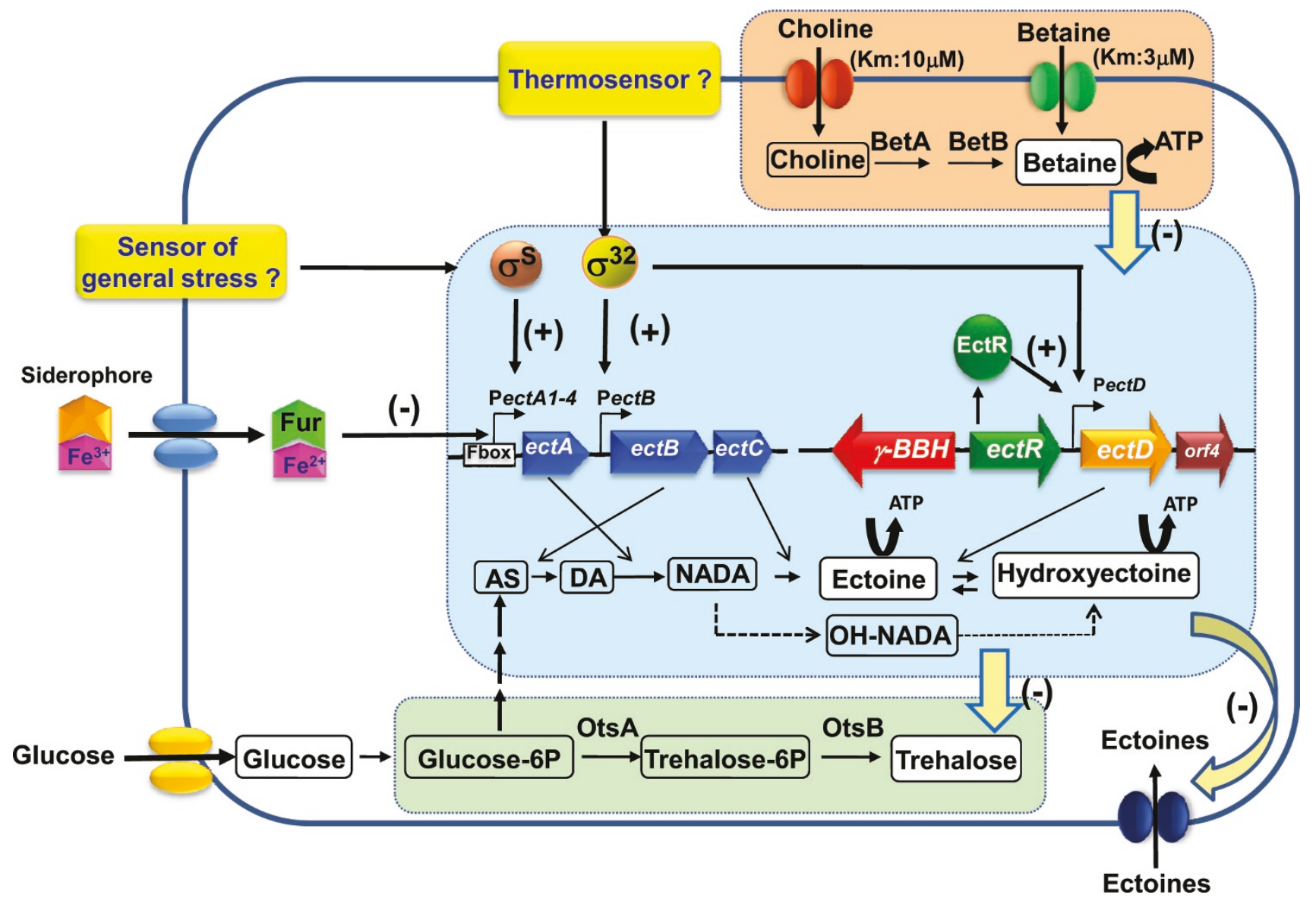

\section{Figure I}

Hierarchical accumulation of the compatible solutes betaine, ectoine, hydroxyectoine and trehalose by Chromohalobacter salexigens. Betaine is accumulated by transport or after choline oxidation. Ectoines and trehalose are synthesized de novo. Betaine inhibits ectoine(s) synthesis and ectoine(s) suppress trehalose accumulation. In addition to osmoprotectants, betaine, its precursor choline, and ectoines, can be used as carbon sources (this has been represented by a curved arrows plus ATP close to ectoine, hydroxyectoine and betaine). The central scheme summarizes the present knowledge on the transcriptional mechanisms governing ectoine(s) synthesis. Abbreviations: AS, L-aspartate $\beta$-semialdehyde; $\gamma$-BBH, $\gamma$-butirobetaine hydroxylase; DA, L-2,4-diaminobutyrate; NADA, N $\gamma$-Acetyl-L-2,4-diaminobutyrate; OH-NADA, 3-Hydroxy$\mathrm{N} \gamma$-Acetyl-L-2,4-diaminobutyrate.

them has been analysed at the molecular level. Gene identifications are available from the author on request, but are not included as annotation is in progress and so identifications are subject to change At the physiological level, we have characterized an osmoregulated high-affinity transport system for betaine $(\mathrm{Km}=3.06 \mu \mathrm{M}$, Fig. 1$)$, which can also take up ectoine and proline betaine (although with less affinity than for betaine), but not choline, choline-O-sulfate or proline [18]. Since proline can be used as a carbon source for $C$. salexigens, and choline or choline-O-sulfate exert osmoprotective effects, we predicted that $C$. salexigens should possess additional transport systems for these solutes [18]. Within the $C$. salexigens genome, there are at least five genes encoding putative BCCT-type (Betaine/Carnitine/Choline-Transport) transporters orthologs to the BetT system of $E$. coli [25] (none of them linked to the betIBA operon encoding choline oxidation), two genes encoding putative $\mathrm{Na}^{+} / \mathrm{pro}^{-}$ line symporters, and five clusters of genes encoding $\mathrm{ABC}$ type betaine/proline uptake systems. This high abundance of osmoprotectant transport systems may support the exquisite adaptation of C. salexigens to hyperosmotic environments.

The betIBA genes, which are responsible for the oxidation of choline into betaine, were isolated and their molecular genetics characterized by our team, in collaboration with Erhard Bremer and co-workers (Fig. 1). Remarkably, BetA 
is able to oxidize both choline and betaine aldehyde and therefore can mediate both steps in the synthesis of betaine [24].

As described before, if present in the surrounding medium, choline-O-sulfate confers osmoprotection [18], but currently it is unknown if choline-O-sulfate is transformed into betaine (via choline, by means of a choline sulphatase activity) or accumulated per se as an osmoprotectant. While the absence, within the C. salexigens genome, of an ortholog to known bet $C$ genes (encoding the choline sulphatase) suggests the second option, this needs experimental confirmation.

Ectoine and hydroxyectoine, the main compatible solutes accumulated by $C$. salexigens in response to salinity [22] can also be taken up from the external medium via at least one transport system whose activity is maximal at optimal salinity (1.5 M NaCl), and shows 3- and 1.5-fold lower values at 0.75 and $2.5 \mathrm{M} \mathrm{NaCl}$, respectively [26]. In contrast, accumulation of betaine by transport from the external medium gradually increases in response to salinity $[18,26]$. At optimal salinity, the rate of ectoine transport is double that of betaine. Very interestingly, the ectA strain CHR62, which cannot synthesize ectoines, showed a 6.8fold increased transport rate if compared with the wild type grown at the same salinity. This result suggests that endogenous ectoine(s) may, directly or indirectly, repress its own transport [26]. C. salexigens genome carries orthologs to the $H$. elongata tea $A B C$ genes (encoding an osmoregulated TRAP-T-type transport system for ectoines [27], but arranged differently (teaA in one strand, followed by teaBC in the opposite strand), and also one ortholog to the Marinococcus halophilus ectM gene for ectoine transport [28]. The characterization of mutants affected in these genes, currently in progress in our laboratory, will give us a clue of which of them is more important for ectoine(s) transport in C. salexigens.

\section{Catabolism of ectoines}

In addition to osmoprotection, C. salexigens can use proline, choline, betaine [18], ectoine and hydroxyectoine [26] as the sole carbon source, although glucose is the preferred carbon source at any salinity. In agreement with this, glucose partially represses, but does not totally abolish, ectoine catabolism [26]. Ectoine and hydroxyectoine support growth only at optimal salinity $(1.5 \mathrm{M} \mathrm{NaCl})$ but not at low $(0.75 \mathrm{M})$ or high $(2.5 \mathrm{M})$ salt. This finding may be correlated with the lower transport rates found at suboptimal salinities [26] and/or control mechanisms repressing ectoine catabolism at high salt, in favour of ectoine(s) accumulation. Within the C. salexigens genome sequence we have found a cluster of 11 genes, all oriented in the same direction, which may encode orthologs to EutB, EutC, EutD, and EutE proteins of Sinorhizobium meliloti, involved in ectoine degradation [29]. However, we found no hits against EutA, and the eutBCDE genes were organized differently, and apart from the ehu ectoine transport system found in S. meliloti. Although this finding suggests that $S$. meliloti and C. salexigens might use the same catabolic routes for ectoines, this requires experimental evidence.

\section{Synthesis of ectoines}

Ectoine and hydroxyectoine are synthesized from aspartate semialdehyde, an intermediate in the biosynthetic route of amino acids derived from aspartic acid (Fig. 2). The biosynthesis of ectoine occurs in three enzymatic steps [30]. First, aspartate semialdehyde is converted into diaminobutyric acid (DA), which is subsequently acetylated to $\mathrm{N} \gamma$-acetyldiaminobutyrate (NADA). The cyclic condensation of this compound leads to the formation of ectoine. Hydroxyectoine is synthesized via ectoine hydroxylation, which is a reversible reaction [31]. On the other hand, we have suggested that C. salexigens can synthesize hydroxyectoine by an alternative pathway that converts $\mathrm{N} \gamma$-acetyldiaminobutyric into hydroxyectoine without the involvement of ectoine [31] (Fig. 2).

The ectoine synthesis genes (ect $A, e c t B$, and $e c t C)$ are very conserved among ectoine-producing bacteria. In C. salexigens, they lay within a 2.8 -kb region encoding the diaminobutyric acid acetyltransferase (EctA), diaminobutyric acid transaminase (EctB), and ectoine synthase (EctC), respectively [32] (Fig. 3). In addition, we have recently isolated and characterized the ectD gene, encoding an ectoine hydroxylase that synthesizes hydroxyectoine from ectoine [33]. ectD is preceded by a regulatory gene (preliminarily named $e c t R$ ), which may encode a transcriptional activator of the ectoine hydroxylase gene (ReinaBueno et al., unpublished results). C. salexigens genome contains a second copy ectD, which has been named ectE, whose expression is negligible if compared to that of ectD (Reina-Bueno et al., unpublished results) (Fig. 3). Moreover, our finding that hydroxyectoine accumulation is drastically reduced in the ectD insertion mutants strongly indicates that ectD is principally responsible for hydroxyectoine synthesis in C. salexigens [33]. Whether or not the remaining hydroxyectoine comes from the activity of EctE or from the proposed alternative route is currently under investigation in our laboratory.

The genetic regions involved in ectoine (ect $A B C)$ and hydroxyectoine (ectD, ectE) synthesis in C. salexigens lay apart within the C. salexigens genome. This organization differs from that found in the sequenced genomes of other ectoines-producing bacteria (all belonging to the Firmicutes, Actinobacteria and Proteobacteria), where different genomic contexts can be found. These include from a complete cluster carrying the ectABC-asK-ectD genes (asK 

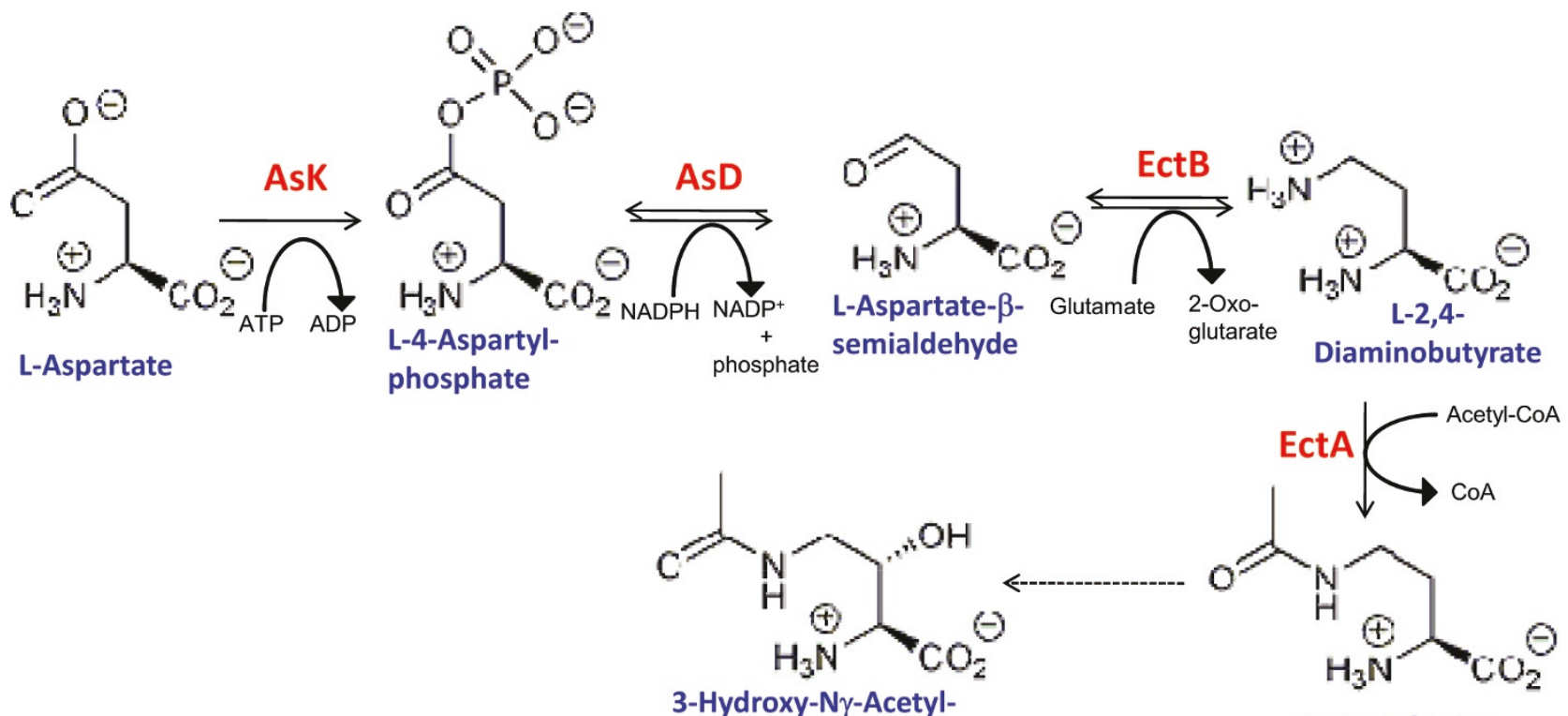

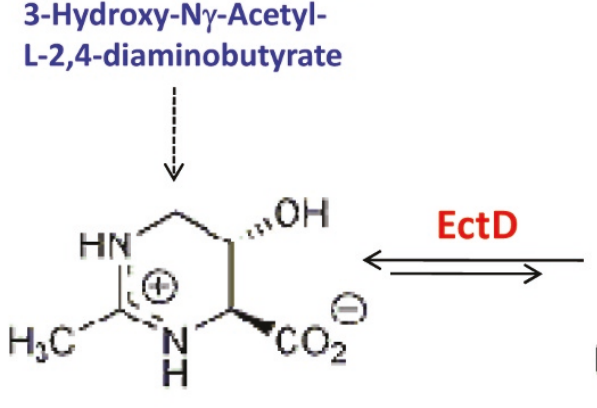

Hydroxyectoine

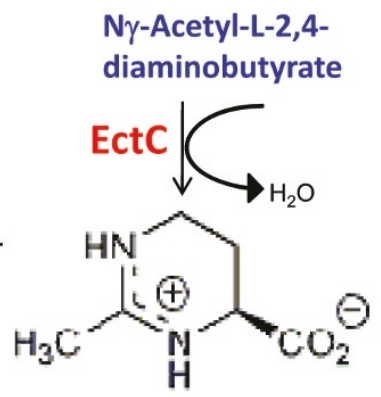

Ectoine

\section{Figure 2}

Biosynthetic pathway for ectoines from aspartate in C. salexigens. The solid and dashed lines indicate established and proposed steps, respectively. The enzymes involved are Aspartate kinase (Ask), Aspartate semialdehyde dehydrogenase (Asd), L-diaminobutyric acid transaminase (EctB), L-diaminobutyric acid acetyl transferase (EctA), ectoine synthase (EctC), and ectoine hydroxylase (EctD).

encoding the aspartate kinase) of "Methylomicrobium alcaliphilum 20Z" (maybe the closest to the ancestral organization) to the set ect $A B C D$ of some Actinobacteria and Proteobacteria, or the scattering of the genes within the chromosome, with duplications of ectC and ectD (Fig. 3). Thus, the general assumption that the organization of the ectoine synthesis genes is very well conserved (ectABC) should be taken with caution, as when more genomes are sequenced, more variations in this genetic organization may be found.

\section{Response of $\mathbf{C}$. salexigens to hypersaline stress}

At the optimal growth temperature $\left(37^{\circ} \mathrm{C}\right)$ and in absence of any osmoprotectant, ectoine, followed by hydroxyectoine, and glutamate and glutamine, are the main compatible solutes accumulated by $C$. salexigens at any salinity (from 0.5 to $3 \mathrm{M} \mathrm{NaCl}$ ) in $\mathrm{M} 63$ minimal medium, as shown by ${ }^{13} \mathrm{C}-\mathrm{NMR}$ analysis $[22,32,33]$. Low levels of alanine, trehalose and lactate can also accumulate as minor solutes in these conditions. When ectoines are quantified by HPLC, their accumulation is maximal during stationary phase of growth. Higher levels of ectoine are found at any salinity tested, and there is an evident increase of the content of both compatible solutes with increasing salt concentration, with 2.75- and 13.8-fold higher levels of ectoine and hydroxyectoine, respectively, in cells grown at $3 \mathrm{M} \mathrm{NaCl}$, if compared to cells grown at $0.75 \mathrm{M} \mathrm{NaCl}$ [33]. Ectoine production is growth-phase dependent in the chloride-dependent Gram-positive halophilic model organism Halobacillus halophilus, which produces ectoine predominantly at very high salinities, along with proline [34]. 


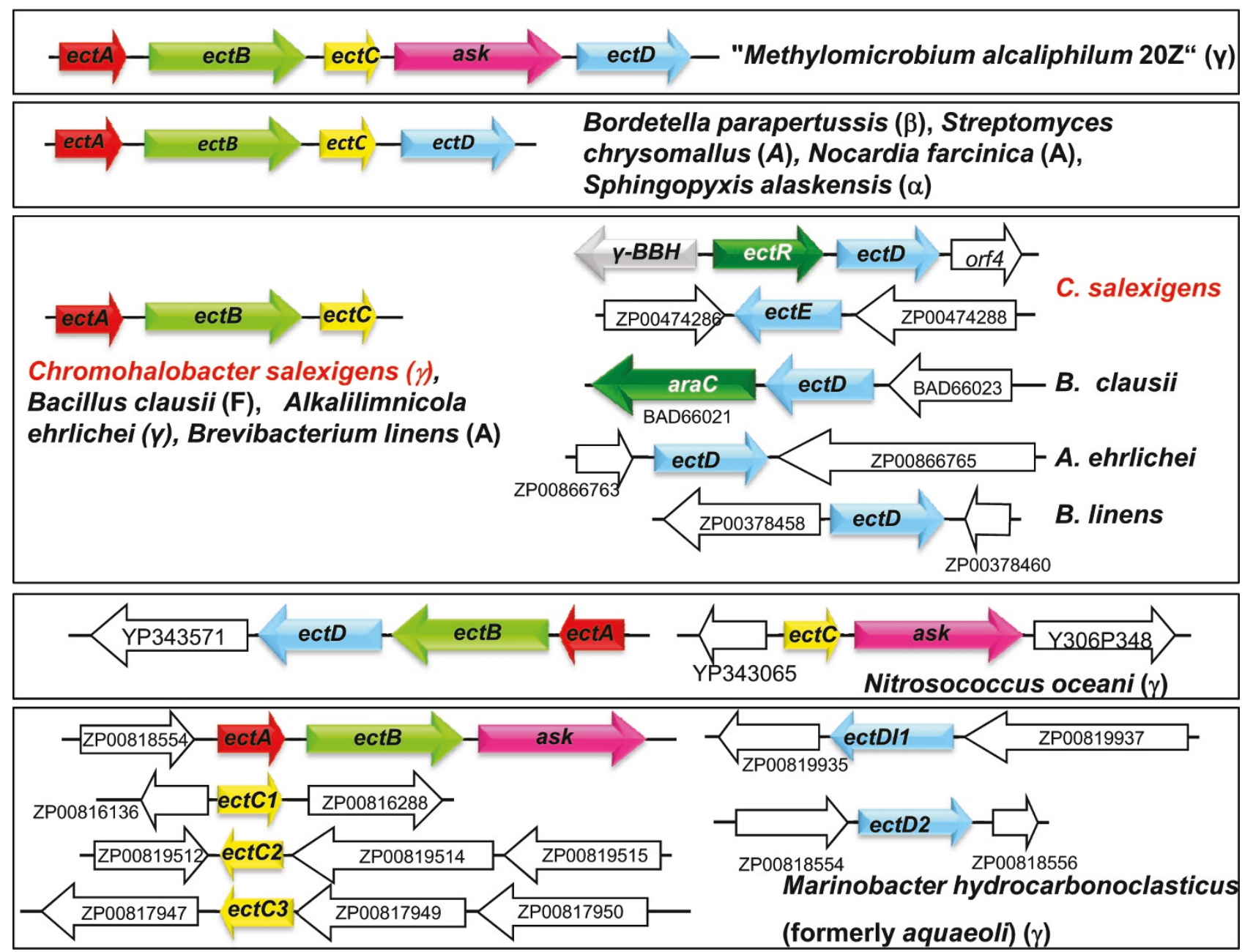

Figure 3

Genomic context of the genes for ectoine (ectABC) and hydroxyectoine (ectD) synthesis in a number of ectoine(s) producing bacteria. Only bacteria whose genome sequence is available are included. The gene ect $E$ of $C$. salexigens encodes a second putative ectoine hydroxylase. as $K$ encodes the aspartate kinase and ect $R$ (in $C$. salexigens) and $a r a C$ (in $B$. clausii) refer to transcriptional regulators of the AraC family. The taxonomic position of the species is abbreviated as follows: A, Actinobacteria, F, Firmicute, $\alpha, \beta, \gamma$, Alpha- Beta-, and Gammaproteobacteria, respectively.

\section{Response of C. salexigens to temperature stress}

The ${ }^{13} \mathrm{C}-\mathrm{NMR}$ spectrum of extracts of C. salexigens grown at $45^{\circ} \mathrm{C}$ with $2.5 \mathrm{M} \mathrm{NaCl}$ (the optimal salinity for growth at this temperature) shows the presence of (in this order) hydroxyectoine, ectoine, trehalose, glutamate, and the ectoine precursor, $\mathrm{N} \gamma$-acetyldiaminobutiric acid [33]. By using HPLC techniques, we quantified ectoine and hydroxyectoine accumulation. Ectoine was the predominant solute from 20 to $35^{\circ} \mathrm{C}$, while the hydroxyectoine levels remained very low at these temperatures. However, the content of ectoine decreased and that of hydroxyectoine increased from 35 to $45^{\circ} \mathrm{C}$, resulting in higher levels of hydroxyectoine than that of ectoine in cells grown at $45^{\circ} \mathrm{C}$, although the pool of ectoines remained constant at a given salinity. These data indicate that the accumulation of hydroxyectoine in C. salexigens is up-regulated by temperature [33]. Thus, hydroxyectoine becomes more important for cells grown under high salinity and high temperature conditions. The finding that an ectD mutant is thermosensitive, but not osmosensitive, confirms that the hydroxyectoine synthesized by EctD is essential for thermoprotection of C. salexigens [33].

The observation that $C$. salexigens accumulates trehalose under high temperature conditions was unexpected, as only traces of this solute can be detected in the wild type strain grown at $37^{\circ} \mathrm{C}[22,26,35]$. This compound is a general stress protectant, including heat stress, that is widely 
spread in both prokaryotic and eukaryotic microorganisms [15]. Within the genome of $C$. salexigens, there are orthologs of the ots $A$ and ots $B$ genes, encoding the enzymes for trehalose synthesis from glucose. Trehalose is also accumulated at $37^{\circ} \mathrm{C}$ in the ectoine-deficient mutant CHR62 [18] indicating that ectoine suppresses, either directly or indirectly, trehalose synthesis in the wild type strain (Fig. 1).

\section{Regulation of the synthesis of ectoines}

In recent years, we have accumulated increasing evidence that ectoine(s) synthesis is a highly controlled process, integrated in the cellular regulatory network in response to (at least) osmotic and heat stress, and further regulated by extracellular solutes and iron. The elucidation of these intricate mechanisms controlling ectoine(s) synthesis, and therefore osmo- and thermoadaptation of C. salexigens, becomes crucial to generate modified strains improved in ectoine(s) production with prospective industrial use.

As described before, our physiological data indicate that the levels of ectoine and hydroxyectoine are maximal during the stationary phase of growth, and that ectoine accumulation is up-regulated by salinity, whereas hydroxyectoine accumulation is up-regulated by both high salt and high temperature [33]. We have now evidence that this regulation occurs, at least in part, at the transcriptional level (Fig. 1). S1 protection assays and transcriptional fusions with lacZ demonstrated that the ectoine synthesis genes (ectABC) can be expressed from two promoter regions. One is located upstream of ectA and composed of four putative promoters (PectA1-4) and the second one is an internal promoter located upstream of ectB (PectB) [35]. Besides, a transcriptional fusion between the region upstream of ectD and lac $Z$ is expressed in E. coli, indicating the existence of promoter activity (M. Reina-Bueno et al. unpublished results).

Expression of PectA-lacZ, PectB-lacZ [35] and PectD-lacZ (Reina-Bueno et al., unpublished results) transcriptional fusions are maximal during stationary phase of growth, in agreement with our physiological data. In addition PectA, PectB [35] and PectD (unpublished results) show promoter activity at low salinity, suggesting that ectABC and ectD may be partially constitutive systems. PectA expression is osmoregulated (in an E. coli background), and the S1 protection assays suggest that PectA1, PectA3 and PectA4 may be the osmoregulated promoters within the PectA region. Expression of PectA and (especially) PectB is induced by continuous growth at high temperature, and repressed in the presence of osmoprotectants (betaine and ectoine), the DNA gyrase inhibitor nalidixic acid, and an excess of iron (only PectA) [35]. Finally, PectD expression is osmoregulated and thermoregulated, as suggested by real-time PCR analysis (Reina-Bueno et al., unpublished results).

The above data indicate that transcription of ectoine(s) synthesis genes involve multiple promoters which allow the system to be regulated by many environmental factors including salinity, temperature, external osmoprotectants, and iron (Fig. 1). Ensuring appropriate expression to this changing environment needs the involvement of a number of transcription factors belonging to different regulatory pathways. The in silico analysis of the -10 and -35 sequences of the regions upstream of ectA, ectB and ectD showed that PectA1 and PectA2 overlap with putative recognition sites of both the main vegetative factor $\sigma^{70}$ [35] and the iron homeostasis regulator Fur (Argandoña et al., unpublished results), whereas PectA3 is similar to $\sigma^{\mathrm{S}}$ dependent promoters [35], and PectB [35] and PectD (Reina-Bueno et al., unpublished results) may be recognized by the heat stress factor $\sigma^{32}$. In agreement with these predictions, expression of PectA-lacZ and PectD-lacZ fusions depend partially on the general stress factor $\sigma^{S}$ and the specific heat stress factor $\sigma^{32}$, respectively, at least in an in E. coli background [35] (Reina-Bueno et al., unpublished results). Both $C$. salexigens and $E$. coli belong to the Gammaproteobacteria, and their $\sigma^{S}$ and $\sigma^{32}$ proteins show a high degree of similarity, suggesting that they could play similar roles in both bacteria.

An additional element involved in the transcriptional control of ectoine hydroxylation seems to be the product of the gene ectR, located upstream of ectD (Figs 1 and 3). We have found that a $C$. salexigens ectR strain grown under high salt and temperature conditions accumulates less hydroxyectoine if compared to the wild type strain (ReinaBueno et al., unpublished results). These data suggest that EctR is a transcriptional activator that might interact with the promoter region upstream of ectD.

Although temperature induction of PectA and PectB expression initially suggested that, in addition to osmoprotection, ectoine might have a physiological role in thermoprotection of C. salexigens [35], we later found that ectoine levels do not increase in response to temperature [33]. One explanation for this is that at high temperature ectoine is rapidly converted to hydroxyectoine by $C$. salexigens and therefore ectoine accumulation decreases in response to temperature. This is compatible with temperature induction of the ectoine synthesis genes, since ectoine is the precursor of hydroxyectoine.

In agreement with the general assumption that transport of compatible solutes is preferred over the synthesis, because the latter is energetically less favourable to the cells [36], C. salexigens cells growing with betaine do not accumulate ectoine(s) at any salinity tested [26,35]. As 
betaine only represses partially the expression of PectA and $P e c t B$, the existence of a post-transcriptional control mechanism that might operate at the level of enzyme activity is inferred (Fig. 1). However, other alternative mechanisms such as an increase efflux of ectoine in the presence of betaine cannot be ruled out.

\section{Conclusion}

C. salexigens needs a fine tuning of its cytoplasmic compatible solute pool in order to cope with a number of abiotic stresses, including high salinity and supra-optimal temperatures. This is achieved by a highly hierarchical accumulation of solutes, starting with external osmoprotectants such as betaine, which totally suppress ectoine(s) synthesis, and followed by endogenous solutes, mainly ectoine and hydroxyectoine. In turn, ectoine(s) inhibit trehalose synthesis, but this is derepressed in response to heat stress. Ectoine and hydroxyectoine are essential for osmoprotection and thermoprotection, respectively. Thus, the traditional role attributed to ectoines as osmoprotectants has been now expanded to protection against other abiotic stresses such as high temperature, or their use as nutrients. In addition to salt and heat stress, ectoine synthesis is transcriptionally modulated by an excess of iron or external osmoprotectants. All these findings, while raising our interest to elucidate the mechanisms governing the synthesis of ectoines, suggest the existence of multiple signal transduction pathways, controlled by general (i.e. $\sigma^{\mathrm{S}}, \sigma^{32}$, Fur) or specific (i.e. EctR) regulators, and predict the need of additional tools (i.e. wide expression analysis, mathematical modelling) in order to achieve a global comprehension of how the whole system is regulated.

\section{Competing interests}

The authors declare that they have no competing interests.

\section{Authors' contributions}

CV and JJN conceived the study, participated in its design and drafted the manuscript. MA, MRB, JRM and CFA have made substantial contributions to acquisition, analysis and interpretation of data concerning uptake of osmoprotectants (JRM), analysis of C. salexigens genome (MA, JRM), characterization of ectoine (CFA) and hydroxyectoine (MA, MRB) synthesis genes, and regulatory studies (MA, MRB). All authors read and approved the manuscript.

\section{Acknowledgements}

This research was financially supported by grants from the European Community (Contract INCO-CT-2004-509I I5), the Spanish Ministerio de Educación y Ciencia (Project BIO2005-06343-CO2-0I), and Junta de Andalucía. Mercedes Reina-Bueno, Javier Rodríguez-Moya and Cristina Fernández-Aunión were recipients of fellowships from Ministerio de Educación y Ciencia (Spain).

\section{References}

I. Csonka LN, Epstein W: Osmoregulation. In Escherichia coli and Salmonella: Cellular and Molecular Biology 2nd edition. Edited by: Neidhart FC, Curtiss JJ. Washington, DC, ASM Press; 1996:1210-1223.

2. ÖByrne $C P$, Booth IR: Osmoregulation and its importance to food-borne microorganisms. Int J Food Microbiol 2002, 74:203-216.

3. Galinski EA, Trüper HG: Microbial behaviour in salt-stressed ecosystems. FEMS Microbiol Rev 1994, I 5:95-108.

4. Bremer E, Krämer R: Coping with osmotic challenges: osmoregulation through accumulation and release of compatible solutes in bacteria. In Bacterial stress responses Edited by: Storz G, Hengge-Aronis R. Washington, D.C., ASM Press; 2000:77-97.

5. Roberts MF: Osmoadaptation and osmoregulation in archaea. Front Biosci 2004, 9:1999-2019.

6. Burg $M$, Kwon E, Kültz D: Regulation of gene expression by hypertonicity. Annu Rev Physiol 1997, 59:437-455.

7. Galinski EA: Osmoadaptation in bacteria. Adv Microb Physiol 1995, 37:272-328.

8. da Costa MS, Santos H, Galinski EA: An overview of the role and diversity of compatible solutes in Bacteria and Archaea. Adv Biochem Eng Biotechnol 1998, 61:1 17-153.

9. Roberts MF: Organic compatible solutes of halotolerant and halophilic microorganisms. Saline Systems 2005:5.

10. Welch WJ, Brown CR: Influence of molecular and chemical chaperones on protein folding. Cell Stress Chaperones 1996, I:I09-I I5.

II. Santos H, da Costa MS: Compatible solutes of organisms that live in hot saline environments. Environ Microbiol 2002, 4:501-509.

12. Yancey $\mathrm{PH}$ : Organic osmolytes as compatible, metabolic and counteracting cytoprotectants in high osmolarity and other stresses. J Exp Biol 2005, 208:2819-2830.

13. Margesin R, Schinner F: Potential of halotolerant and halophilic microorganisms for biotechnology. Extremophiles 200I, 5:73-83.

14. Lentzen G, Schwartz T: Extremolytes: Natural compounds from extremophiles for versatile applications. Appl Microbiol Biotechnol 2006, 72:623-634.

15. Welsch DT: Ecological significance of compatible solute accumulation by micro-organisms: from single cells to global climate. FEMS Microbiol Rev 2000, 24:263-290.

16. Ventosa A, Nieto JJ, Oren A: Biology of moderately halophilic aerobic bacteria. Microbiol Mol Biol Rev 1998, 62:504-544.

17. Arahal D, García MT, Vargas C, Cánovas D, Nieto JJ, Ventosa A: Chromohalobacter salexigens sp. nov., a moderately halophilic species that includes Halomonas elongata DSM 3043 and ATCC 33 I 74. Int J Syst Evol Microbiol 200 I, 5 I (Pt 4): I 457-I 462.

18. Cánovas D, Vargas C, Csonka LN, Ventosa A, Nieto Jj: Osmoprotectants in Halomonas elongata: high-affinity betaine transport system and choline-betaine pathway. J Bacteriol 1996, I 78:722|-7226.

19. O'Connor K, Csonka LN: The high salt requirement of the moderate halophile Chromohalobacter salexigens DSM3043 can be met not only by $\mathrm{NaCl}$ but by other ions. Appl Environ Microbiol 2003, 69:6334-6336.

20. Vargas C, Nieto J]: Genetic tools for the manipulation of moderately halophilic bacteria of the family Halomonadaceae. In Methods in Molecular Biology, Recombinant Gene Expression: Reviews and Protocols Volume 267. 2nd edition. Edited by: Balbas P, Lorence A. Totowa, NJ, Humana Press; 2004: I83-208.

21. Vargas C, Kallimanis A, Koukkou Al, Calderón MI, Cánovas D, Iglesias-Guerra F, Drainas C, Ventosa A, Nieto JJ: Contribution of chemical changes in membrane lipids to the osmoadaptation of the halophilic bacterium Chromohalobacter salexigens. Syst Appl Microbiol 2005, 28:571-58I.

22. Cánovas D, Vargas C, Iglesias-Guerra F, Csonka LN, Rhodes D, Ventosa A, Nieto J]: Isolation and characterization of salt-sensitive mutants of the moderate halophile Halomonas elongata and cloning of the ectoine synthesis genes. J Biol Chem 1997, 272:25794-2580l.

23. Cánovas D, Vargas C, Csonka LN, Ventosa A, Nieto Jj: Synthesis of glycine betaine from exogenous choline in the moderately halophilic bacterium Halomonas elongata. Appl Environ Microbiol 1998, 64:4095-4097. 
24. Cánovas D, Vargas C, Kneip S, Morón MJ, Ventosa A, Bremer E, Nieto J): Characterization of the genes for the synthesis of the compatible solute glycine betaine in the moderately halophilic bacterium Halomonas elongata DSM 3043. Microbiology 2000, I 46:455-463.

25. Lamark T, Kaasen I, Eshoo MW, Falkenberg P, McDougall J, Strøm AR: DNA sequence and analysis of the bet genes encoding the osmoregulatory choline-glycine betaine pathway of Escherichia coli. Mol Microbiol 199I, 5: 1049-64.

26. Vargas C, Jebbar M, Carrasco R, Blanco C, Calderón MI, IglesiasGuerra F, Nieto Jj: Ectoines as compatible solutes and carbon and energy sources for the halophilic bacterium Chromohalobacter salexigens. J Appl Microbiol 2006, 100:98-107.

27. Grammann K, Volke A, Kunte HJ: New type of osmoregulated solute transporter identified in halophilic members of the Bacteria domain: TRAP transporter TeaABC mediates uptake of ectoine and hydroxyectoine in Halomonas elongata DSM 258 IT. J Bacteriol 2002, I 84:3078-3085.

28. Vermeulen V, Kunte HJ: Marinococcus halophilus DSM 20408T encodes two transporters for compatible solutes belonging to the betaine-carnitine-choline transporter family: identification and characterization of ectoine transporter EctM and glycine betaine transporter BetM. Extremophiles 2004, 8:175-184.

29. Jebbar M, Sohn-Bösser L, Bremer E, Bernard T, Blanco C: Ectoineinduced proteins in Sinorhizobium meliloti include an ectoine ABC-type transporter involved in osmoprotection and ectoine catabolism. J Bacteriol 2005, 187:1293-1304.

30. Peters $P$, Galinski EA, Trüper HG: The biosynthesis of ectoine. FEMS Microbiol Lett 1990, 71:157-162.

31. Cánovas D, Borges N, Vargas C, Ventosa A, Nieto J], Santos H: Role of $\mathbf{N} \gamma$-acetyldiaminobutirate as an enzyme stabilizer and an intermediate in the biosynthesis of hydroxyectoine. Appl Environ Microbiol 1999, 65:3774-3779.

32. Cánovas D, Vargas C, Calderón MI, Ventosa A, Nieto J]: Characterization of the genes for the biosynthesis of the compatible solute ectoine in the moderately halophilic bacterium Halomonas elongata DSM 3043. Syst Appl Microbiol 1998, 2 I:487-497.

33. García-Estepa R, Argandoña M, Reina-Bueno M, Capote N, IglesiasGuerra F, Nieto JJ, Vargas C: The ectD gene, which is involved in the synthesis of the compatible solute hydroxyectoine, is essential for thermoprotection of the halophilic bacterium Chromohalobacter salexigens. J Bacteriol 2006, 188:3774-3784.

34. Saum SH, Müller V: Growth phase-dependent switch in osmolyte strategy in a moderate halophile: ectoine is a minor osmolyte but major stationary phase solute in Halobacillus halophilus. Environ Microbiol 2008, 10:716-726.

35. Calderón MI, Vargas C, Rojo F, Iglesias-Guerra F, Csonka LN, Ventosa $A$, Nieto JJ: Complex regulation of the synthesis of the compatible solute ectoine in the halophilic bacterium Chromohalobacter salexigens. Microbiology 2004, I 50:305 I-3063.

36. Oren A: Bioenergetic aspects of halophilism. Microbiol Mol Biol $\operatorname{Rev} 1$ 1999, 63(2):343-348.

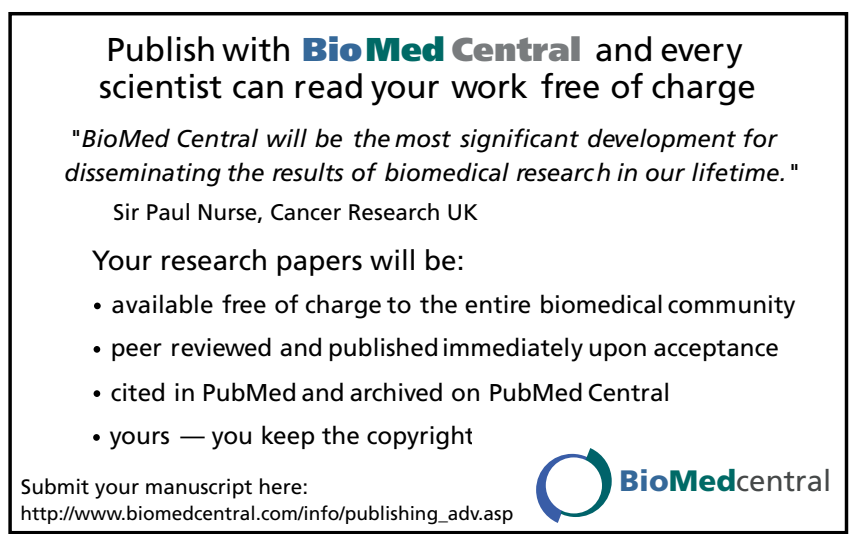

